\title{
Increased chitotriosidase activity in plasma of patients with type 2 diabetes
}

\author{
Ewa Żurawska-Płaksej ${ }^{1}$, Maria Knapik-Kordecka², Anna Rorbach-Dolata ${ }^{3}$, Agnieszka Piwowar ${ }^{3}$
}

${ }^{1}$ Department of Pharmaceutical Biochemistry, Wroclaw Medical University, Poland 2Department and Clinic of Angiology, Hypertension and Diabetology, Wroclaw Medical University, Wroclaw, Poland

${ }^{3}$ Department of Toxicology, Wroclaw Medical University, Wroclaw, Poland

Submitted: 24 July 2014

Accepted: 21 September 2014

Arch Med Sci 2016; 12, 5: 977-984

DOI: 10.5114/aoms.2016.60093

Copyright $\odot 2016$ Termedia \& Banach

\author{
Corresponding author: \\ Ewa Żurawska-Płaksej \\ Department of Pharmaceutical \\ Biochemistry \\ Wroclaw Medical University \\ 211a Borowska St \\ 50-556 Wroclaw, Poland \\ Phone: +48717840468 \\ E-mail: \\ ewa.zurawska-plaksej@ \\ umed.wroc.pl
}

\begin{abstract}
Introduction: Chitotriosidase (CHIT1) is a chitinolytic enzyme involved mainly in the immune and inflammatory response. It shows increased activity in many pathologies, including in newly diagnosed type 2 diabetes (T2D). This study aimed to investigate this enzyme's activity in plasma of patients with ongoing T2D and indicate factors related to the increased activity of this enzyme.

Material and methods: Ninety-one patients and 46 control subjects without abnormalities in carbohydrate metabolism and inflammatory states were enrolled in the study. Plasma CHIT1 activity was measured by a spectrofluorometric method. Routine laboratory parameters such as blood glucose, total cholesterol and HDL fraction, triglyceride, glycated hemoglobin, white blood cell count and C-reactive protein were measured by standard methods. Results: We found that the chitotriosidase activity was significantly higher $(p<0.001)$ in type 2 diabetic patients and positively associated with parameters of glycemic control (levels of glucose and glycated hemoglobin) and blood pressure. Plasma glucose level and systolic blood pressure were independent determinants of increased CHIT1 activity in T2D patients, even after adjustment for disease duration, body mass index, parameters of inflammation and lipid metabolism. We also found that increased CHIT1 activity was associated with occurrence of diabetic angiopathies.

Conclusions: This investigation indicates a possible role of chitotriosidase in the course of T2D, especially in relation to development of diabetic angiopathies.
\end{abstract}

Key words: chitinase, type 2 diabetes, diabetic angiopathies, glucose, systolic blood pressure.

\section{Introduction}

Chitotriosidase (EC 3.2.1.14), also named chitinase-1 (CHIT1), belongs to the 18 family of chitinases and shows hydrolytic activity against chitin. Although vertebrates do not synthesize chitin, the enzyme was in the 1990s unexpectedly discovered in humans [1-3]. The exact function of human CHIT1 remains unclear, but its participation in immune defense is postulated. The enzyme's ability to cleave the $\beta$ - $(1,4)$-glycosidic linkages in chitin determines its antibacterial, antifungal and antiparasitic action [4]. Increased serum CHIT1 activity, resulting from macrophage stimulation, has been recognized as a useful marker in diagnosing and moni- 
toring Gaucher disease [5]. Recently it has been reported that increased activity of chitotriosidase may also be connected with the inflammatory state, and therefore a number of investigations on the role of this enzyme in different pathological units, mainly with inflammatory background, have been carried out [6]. Some authors have postulated participation of chitotriosidase in the development of atherosclerosis, which creates a possible link to the course of type 2 diabetes (T2D) [7].

Type 2 diabetes, the most common form of diabetes, results from insulin resistance and/or deficiency and is connected with many metabolic abnormalities, such as hyperglycemia, obesity, hypertension, and dyslipidemia. These are main systemic factors, which trigger proinflammatory events, leading to endothelial dysfunction and increased prevalence of diabetic angiopathies (DA) in T2D diabetic patients. Diabetic angiopathies are the major cause of decreased quality of life and increased mortality of $\mathrm{T} 2 \mathrm{D}$, remaining a constant target of diagnosis, monitoring and treatment [8-12].

The aim of our study was to investigate plasma CHIT1 activity in patients with ongoing type 2 diabetes and evaluate its possible relationship with anthropometric and metabolic parameters, describing the diabetic state. Moreover, we assessed whether this enzyme could provide predictive information for the risk of occurrence of diabetic angiopathies.

\section{Material and methods}

Initially, 91 patients with type 2 diabetes mellitus, of average duration 11 years, were recruited from the Clinic of Angiology, Hypertension and Diabetology of Wroclaw Medical University. Type 2 diabetes was diagnosed according to the standards set by the Polish Diabetes Association [13]. Diabetic angiopathies were recognized in 44 patients (27\% of them had microangiopathy, $30 \%$ had macroangiopathy and $43 \%$ had both micro- and macroangiopathies). Patients were treated continuously with routine medications (including hypoglycemic, hypotensive and hypolipidemic agents if necessary) for 3 months before examination. The control group consisted of 46 subjects without $a b$ normalities in carbohydrate metabolism (glucose level $<5.5 \mathrm{mmol} / \mathrm{l}$ ) and a history of inflammatory states during the last 3 months, matched for sex and age. All participants were informed about the aim of the study and their written permission was given. The use of human blood was approved by the Local Bioethics Committee of Wroclaw Medical University.

Venous blood was collected after overnight fasting into standard vacuum tubes with heparin $(16 \mathrm{lU} / \mathrm{ml})$ to obtain plasma. Routine biochemical parameters were measured on admission to the hospital by the standard methods using Cobas Mira Plus (ROCHE). Subjects from the control group were examined using the same methods. Plasma samples were stored at $-80^{\circ} \mathrm{C}$ until assayed. To measure chitotriosidase activity a $5 \mu \mathrm{l}$ plasma sample was incubated with $100 \mu \mathrm{l}$ of substrate solution (22 $\mu \mathrm{M}$ 4-methylumbelliferyl- $\beta-N-N$ '-N"-triacetylchitotrioside (Sigma Chemical (C, USA) in Mcllvaine's buffer, $\mathrm{pH}$ 5.0) for $15 \mathrm{~min}$ at $37^{\circ} \mathrm{C}$ (modified from Hollak et al., 1994). The reaction was terminated using $2 \mathrm{ml}$ of $0.3 \mathrm{M}$ glycine- $\mathrm{NaOH}$ buffer $(\mathrm{pH}$ 10.5) and fluorescence of the product, 4-methylumbelliferone, was measured with a Perkin Elmer LS 50B fluorometer at excitation $\lambda=365 \mathrm{~nm}$ and emission $\lambda=445 \mathrm{~nm}$. Intra- and interassay coefficients of variation were $4.9 \%$ and $5.3 \%$, respectively. Thirteen participants with CHIT1 activity at zero level were excluded from further analysis. Finally, 82 patients and 42 control subjects were evaluated, and their anthropometric and clinical features are presented in Table I.

Participants were divided into subgroups based on: a) body mass index (BMI $\geq 30 \mathrm{~kg} / \mathrm{m}^{2}$ - obese, $\geq 25 \mathrm{~kg} / \mathrm{m}^{2}$ and $\leq 29.9 \mathrm{~kg} / \mathrm{m}^{2}$ - overweight, $\leq 24.9 \mathrm{~kg} /$ $\mathrm{m}^{2}$ - normal weight), b) presence of dyslipidemia (defined as total cholesterol $\geq 4.9 \mathrm{mmol} / \mathrm{l}$ and/or LDL cholesterol (LDL-Ch) $\geq 3.4 \mathrm{mmol} / /$ or $\mathrm{HDL}$ cholesterol (HDL-Ch) $<1.0 \mathrm{mmol} / \mathrm{l}$ in males and $<1.3 \mathrm{mmol} / \mathrm{l}$ in females or triglyceride (TG) $\geq 1.7 \mathrm{mmol} / \mathrm{l}), \mathrm{c}$ ) quality of short-term glycemic control (plasma glucose $\geq 7 \mathrm{mmol} / \mathrm{l}$ ), d) blood pressure control ( $\geq 140 / 90$ ), e) presence of inflammation (recognized if white blood cell (WBC) count $\geq 10.9 \times 10^{9} / \mathrm{l}$ or C-reactive protein (CRP) concentration > $6 \mathrm{mg} / \mathrm{l})$, f) smoking status [13, 14]. Based on the pharmacological treatment of hyperglycemia, the patients were subdivided into those treated with insulin alone $(n=21)$ and those treated with insulin and oral agents (mainly metformin, $n=61$ ).

\section{Statistical analysis}

The statistical analysis was performed using Statistica PL for Windows, version 10.0. The nonparametric Mann-Whitney $U$ test was used for the comparison between continuous variables and the $\chi^{2}$ test for categorical variables. Univariate and multiple regression analysis was performed to evaluate the association of CHIT1 activity (as the dependent variable) with the explanatory variables in the examined population. Stepwise logistic linear regression was used to evaluate the predictive value of the increased CHIT1 levels (as the independent variable). A p-value below 0.05 was considered statistically significant. All results were expressed as a mean value and a standard deviation. 
Table I. Anthropometric and clinical characteristics of participants

\begin{tabular}{|c|c|c|}
\hline Parameter & Diabetic subjects & Control subjects \\
\hline$N$ (male/female) & $82(38 / 44)$ & $42(18 / 24)$ \\
\hline Age [years] & $59.73 \pm 5.58$ & $57.37 \pm 5.51$ \\
\hline Disease duration [years] & $11.01 \pm 5.13$ & - \\
\hline $\mathrm{BMI}\left[\mathrm{kg} / \mathrm{m}^{2}\right]$ & $30.23 \pm 4.51$ & $27.75 \pm 3.91^{*}$ \\
\hline $\mathrm{SBP}[\mathrm{mm} \mathrm{Hg}]$ & $140.00 \pm 14.00$ & $122.00 \pm 11.00^{* * *}$ \\
\hline $\mathrm{DBP}[\mathrm{mm} \mathrm{Hg}]$ & $80.50 \pm 9.11$ & $76.50 \pm 8.50$ \\
\hline $\mathrm{CRP}[\mathrm{mg} / \mathrm{l}]$ & $10.38 \pm 9.41$ & $4.4 \pm 3.49^{* * *}$ \\
\hline WBC $\left[\times 10^{9} / 1\right]$ & $7.64 \pm 4.91$ & $7.05 \pm 1.75^{\star * *}$ \\
\hline Glucose $[\mathrm{mmol} / \mathrm{l}]$ & $8.93 \pm 2.91$ & $5.00 \pm 0.72^{* * *}$ \\
\hline $\mathrm{HbA}_{1 \mathrm{c}}(\%)$ & $7.8 \pm 1.54$ & $5.67 \pm 0.27^{\star \star *}$ \\
\hline Total-Ch $[\mathrm{mmol} / \mathrm{l}]$ & $5.08 \pm 1.07$ & $5.19 \pm 2.36^{*}$ \\
\hline $\mathrm{HDL}-\mathrm{Ch}[\mathrm{mmol} / \mathrm{l}]$ & $1.16 \pm 0.29$ & $1.45 \pm 0.38^{* *}$ \\
\hline LDL-Ch $[\mathrm{mmol} / \mathrm{l}]$ & $2.95 \pm 0.85$ & $2.95 \pm 0.61$ \\
\hline $\mathrm{TG}[\mathrm{mmol} / \mathrm{l}]$ & $2.34 \pm 0.81$ & $1.56 \pm 0.38^{\star * *}$ \\
\hline Smoking (\%) & 10 & 12 \\
\hline
\end{tabular}

Data are presented as mean \pm standard deviation or number/percent, statistical significance: ${ }^{*} p<0.5,{ }^{* *} p<0.01,{ }^{* * *} p<0.001$, $B M I$ - body mass index, SBP - systolic blood pressure, DBP - diastolic blood pressure, CRP - C-reactive protein, WBC - white blood cells, $H_{b A_{1 c}}$ - glycated hemoglobin, total-Ch - total cholesterol, HDL-Ch - HDL cholesterol, LDL-Ch - LDL cholesterol, TG - triglyceride.

\section{Results}

Plasma chitotriosidase activities in patients with type 2 diabetes mellitus and control subjects are presented in Figure 1 (first two bars). A statistically significant $(p<0.001)$ increase of CHIT1 activity (over 70\%) was observed in type 2 diabetic patients compared with the control subjects. No significant differences in CHIT1 activity were observed in subgroups of diabetic patients divided according to BMI value, as well as in subgroups created on the basis of presence of dyslipidemia and inflammation. Neither tobacco use nor ap-

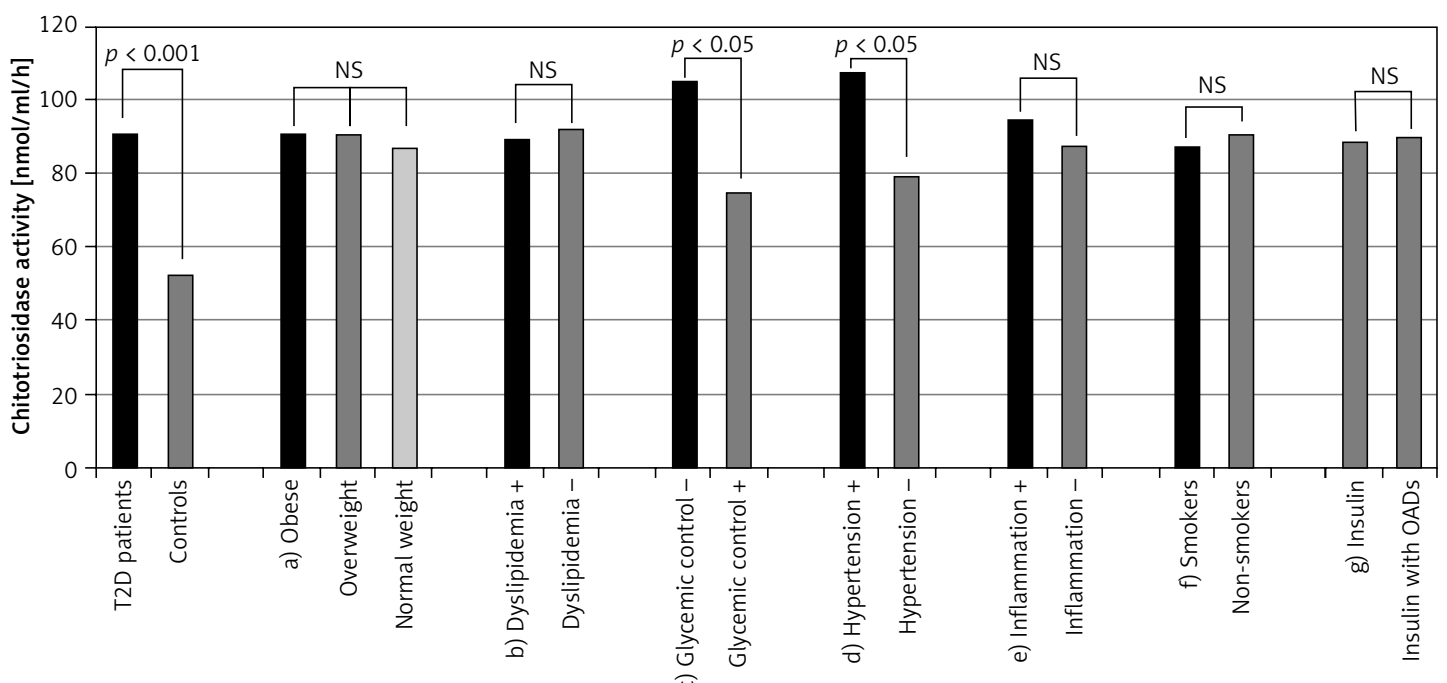

Figure 1. Chitotriosidase activity in examined population and in different subgroups of diabetic patients. First two bars illustrate activity of chitotriosidase in all patients with type 2 diabetes and controls. Next bars illustrate activity of chitotriosidase in distinguished subgroups of diabetic patients: a) patients with obesity, overweight and normal weight, b) patients with and without dyslipidemia, c) patients with poor and good short-term glycemic control, d) patients with poorly controlled hypertension and normalized blood pressure, e) patients with and without symptoms of inflammation, f) smokers and non-smokers, g) patients treated with insulin or insulin with oral antidiabetic drugs (OADs); NS - not statistically significant 
plied hypoglycemic treatment had an influence on activity of this enzyme.

To identify the factors associated with increased CHIT1 activity in examined participants (divided or not according to presence of T2D) univariate regression analysis was performed at first, and the results are shown in Table II. Plasma CHIT1 activity was the dependent variable, and the explanatory variables were duration of diabetes, obesity (described by BMI value), parameters of glycemic control (glucose and glycated hemoglobin), inflammation (CRP and WBC), lipid metabolism (HDL-Ch, LDL-Ch and TG) and systolic and diastolic blood pressure (SBP, DBP). In the total population CHIT1 activity was significantly associated with plasma glucose and HDL-Ch concentration, SBP and DBP, whereas in type 2 diabetic patients CHIT1 activity was additionally associated with glycated hemoglobin $\left(\mathrm{HbA}_{1 \mathrm{c}}\right)$, but not with HDL-Ch. However, in the control group CHIT1 activity was associated with CRP and SBP. To further determine the independent factors associated with the increased plasma CHIT1 activity, multiple regression analysis was performed, and the results are given in Table III. Body mass index, plasma glucose, $\mathrm{HbA}_{1}$, CRP, SBP, DBP, LDL-Ch, HDL$\mathrm{Ch}$ and TG were included in the analyses, provided that the $p$ value was below 0.1 in the univariate model. In the present study we focused on T2D patients. As shown in Table III, only plasma glucose and SBP were still independent predictors of increased plasma CHIT1 activity in T2D patients, and $14 \%$ of the variance of CHIT1 activity could be predicted by these variables. Plasma glucose has stronger predictive value $(\beta=0.37, p<0.01)$ than $\operatorname{SBP}(\beta=0.27, p<0.05)$.

As the last step, we assessed whether the increased CHIT1 activity may predispose to occurrence of diabetic angiopathies. CHIT1 activity was transformed into categorical data, according to the mean value $(52.3 \mathrm{nmol} / \mathrm{ml} / \mathrm{h})$ observed in the control group, also corresponding to the first quartile of enzyme activity in diabetic patients. Values equal to or above $52.3 \mathrm{nmol} / \mathrm{ml} / \mathrm{h}$ were considered as increased enzyme activity (signed as yes $=1$ ). Among the individuals with $\mathrm{T} 2 \mathrm{D}$, the proportion of patients with angiopathies was significantly higher in those with CHIT1 activity $\geq 52.3 \mathrm{nmol} / \mathrm{ml} / \mathrm{h}$ (36/43, which is $86 \%$ ), compared to patients with CHIT1 activity $<52.3 \mathrm{nmol} / \mathrm{ml} / \mathrm{h}$ (8/39, which is $20 \%)$. A logistic linear regression analysis with the presence of DA (yes/no) as a dependent variable and CHIT1 activity along with other well-known risk factors as predictors was performed, and the results are presented in Table IV. As revealed in the univariate analysis, increased CHIT1 activity, hyperglycemia, presence of inflammation, hypertension and hypertriglyceridemia were predictors of occurrence of DA. In the multivariate analysis, increased CHIT1 activity remained the independent predictor for occurrence of diabetic angiopathies in the constructed model, and the highest odds ratios were obtained for the presence of inflammation, increased CHIT1 activity and poor shortterm glycemic control.

\section{Discussion}

In the current study we firstly revealed a significant $(p<0.001)$, almost 2 -fold, increase of chitotriosidase activity in plasma of patients with ongoing type 2 diabetes mellitus in comparison to the control group. About $75 \%$ of diabetic patients had higher CHIT1 activity than in the control group, which is not fully explicable. To our knowledge, in the scientific literature only a single study describes CHIT1 activity in patients with newly diagnosed, untreated, and uncomplicated T2D [15]. The authors reported increased activity of chitotriosidase in these patients and its significant correlation with age, plasma glucose level and asymmetric dimethylarginine concentration, although the strength of the correlations was rather weak. On the basis of these findings, the authors postulated the relevance of CHIT1 for endothelial functions and insulin resistance in the examined population and concluded that increased CHIT1 activity may be a predictor of endothelial dysfunction. However, reported associations pertained to the whole examined population (with and without diabetes), and no information was provided about CHIT1 specifically in the group of diabetic patients. Moreover, the influence of other parameters, which may also affect endothelial function, e.g. hypercholesterolemia, hypertension, or inflammation, was not taken into consideration $[8,16,17]$.

In this work, we undertook research to evaluate the influence of metabolic disturbances accompanying diabetes on CHIT1 activity. First of all, we found that neither obesity nor dyslipidemia significantly affected enzyme activity in the examined patients (Figure 1). Regression analysis assessed the lack of relationship between plasma CHIT1 activity and parameters of lipid metabolism in type 2 diabetic patients and revealed that the enzyme activity was not associated with duration of the disease or BMI value. Alanbay et al. [18] also demonstrated that elevation of CHIT1 activity was independent of obesity in women with polycystic ovary syndrome. In contrast to Sonmez et al. [15], we did not find any association with age. It may result from the adjustment for age in our study, although the results obtained by Ramanathan et al. [19] indicate that elevation of CHIT1 activity may be caused by normal age-related chronic macrophage activation, not their acute 


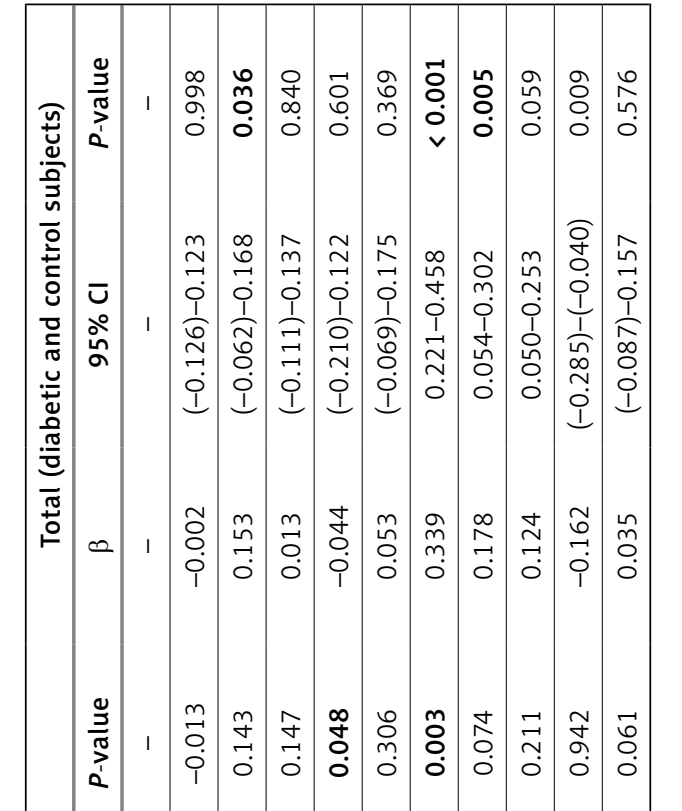

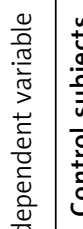

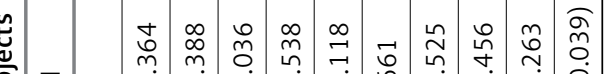

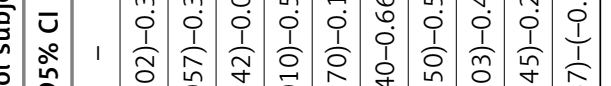

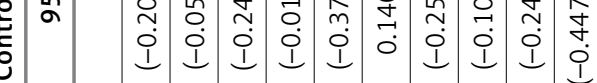

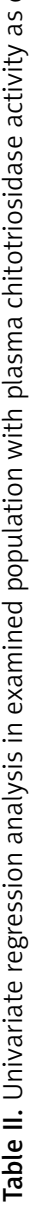

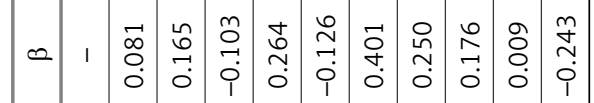

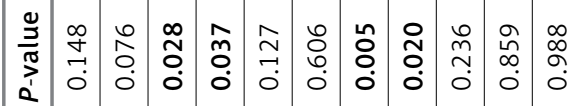

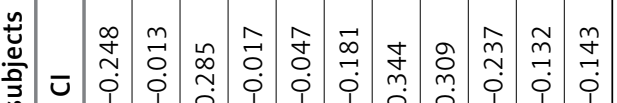

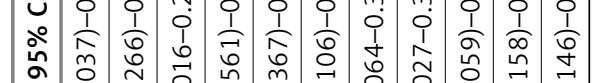

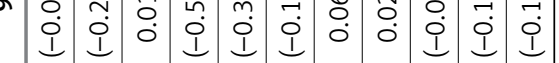

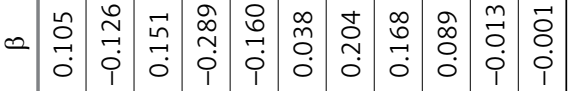

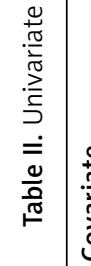

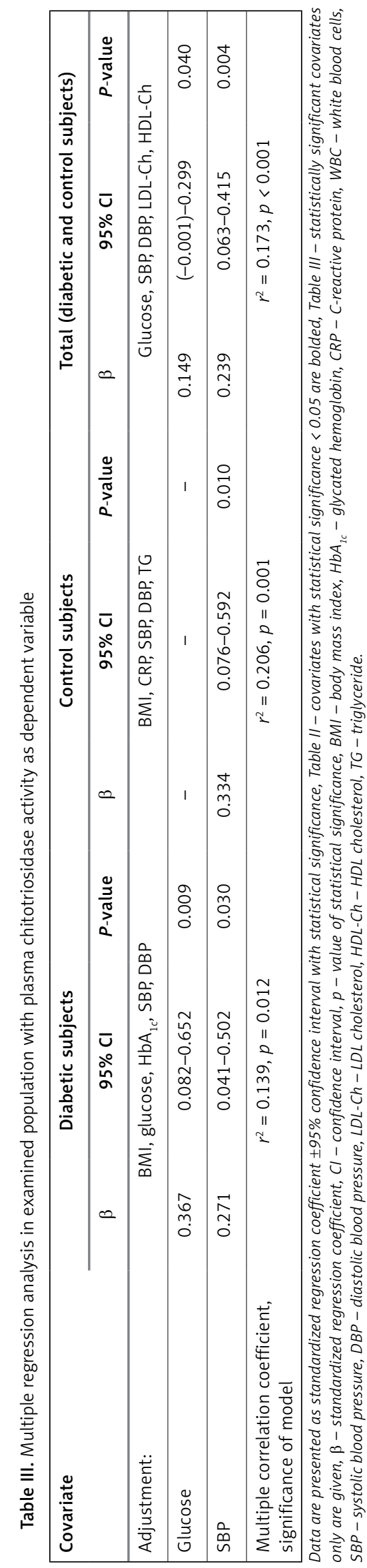

Arch Med Sci 5, October / 2016 


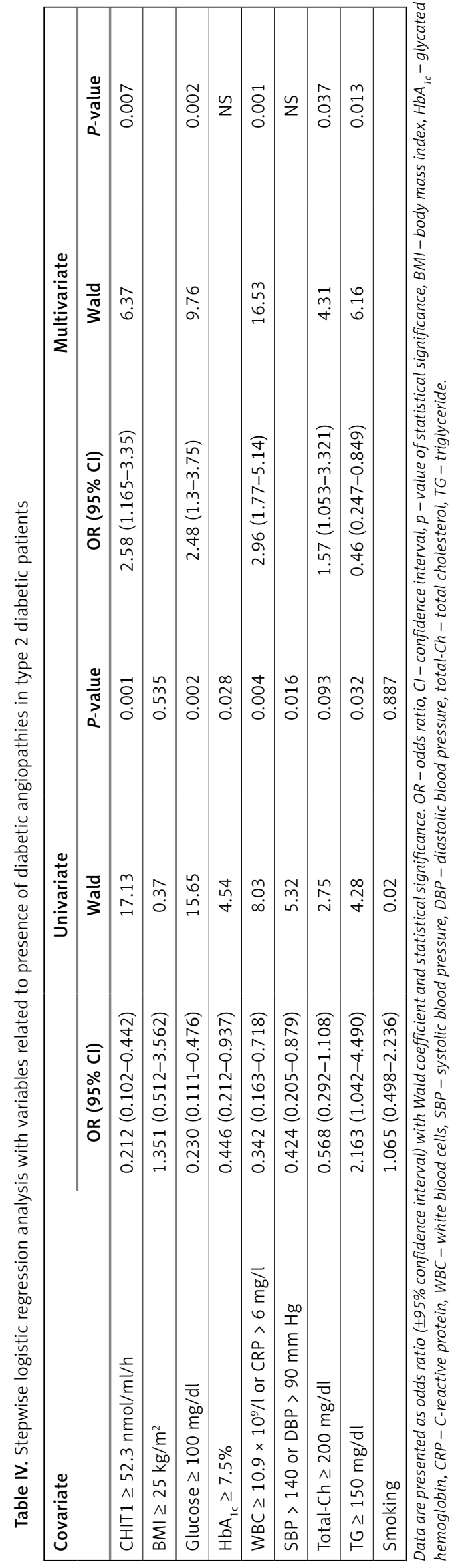

activation or an inflammatory state. A stepwise multiple regression analysis revealed that plasma CHIT1 activity was independently correlated with glucose level in the total population and in diabetic patients, which may indicate its participation in the course of diabetes. We also found that CHIT1 activity was additionally associated with the SBP value in all three analyzed groups (stratified or not according to presence of diabetes). So far, a significant positive correlation of this enzyme's activity and blood pressure (but with DBP) has only been found in pre-eclamptic pregnant women [20]. Since hypertension is a known risk factor for cardiovascular diseases, we may speculate that it may play an important role in the development and/or progression of unfavorable vascular changes, which is linked with increased risk for mortality and morbidity in diabetes. Interestingly, although chitotriosidase has been proposed as an inflammatory marker, we did not find any association with CRP in diabetic patients, which was, by contrast, observed in the control group. So far, a lack of significant correlation between serum CHIT activity and CRP, as well as IL-6, has been reported in several studies concerning obese children and adolescents [21, 22]. Therefore, it may be suggested that CHIT1 activity does not reflect the chronic inflammatory state occurring in type 2 diabetes, but is connected with inflammatory episodes of other etiology.

It is well known that diabetes mellitus is associated with multifaceted metabolic disorders. High prevalence of hypertension and dyslipidemia is demonstrated among patients with type 2 diabetes. Thus, this factors could not be ignored when analyzing various metabolic interplays in the disease course. Hypertension and dyslipidemia (alongside hyperglycemia, obesity and tobacco use) are influential factors for future development of vascular diseases, which lead to failure of various organs, significantly lowering quality of life and shortening the lifespan of diabetic patients $[8,16,23]$. It is important in our study to estimate their influence on chitotriosidase activity in the plasma of type 2 diabetic patients in the context of its possible participation in the development of diabetic angiopathies. Boot et al. [24] firstly demonstrated an association between CHIT1 expression and lipid-laden macrophages in the atherosclerotic vessel wall, and Artieda et al. [7] suggested a role for CHIT1 as a marker of atherosclerotic extension and showed its predictive value in determining the risk of new cardiovascular events in a population with stable coronary heart disease during 4-year follow-up studies. In the present study we found that patients with increased CHIT1 activity had higher prevalence of diabetic angiopathies compared to those with 
enzyme activity similar to the control group. We suggest that chitotriosidase may be another causal factor that contributes to the development of vascular changes in diabetes. Multiple logistic regression analysis shows a relationship between increased CHIT1 activity and occurrence of DA. After adjustment for hyperglycemia, presence of inflammation, hypertension, hypercholesterolemia and hypertriglyceridemia, CHIT1 activity remained independently correlated with the presence of diabetic angiopathies. In the constructed model, the CHIT1 odds ratio for presence of diabetic angiopathies was almost the same as for glucose, which indicates the significance of increased CHIT1 activity in the pathomechanism of DA. However, it is known that there are also other mechanisms which trigger the inflammatory response in T2D. For example, oxidative stress is considered as an important factor that promotes endothelial dysfunction and further contributes to development of vascular abnormalities. As diabetes progresses, other metabolic disturbances also develop, creating a vicious circle of metabolic decline. It is not easy to evaluate whether increased CHIT1 activity is a cause or rather a consequence of DA, but this work shows an evident relationship between these events. Unfortunately, in the present study we did not distinguish subgroups with micro- and macrovascular complications, which could explain whether the observed increase in CHIT1 activity is independent of vessel size and, indirectly, of the pathomechanism of diabetic angiopathies (which is different for micro- and macroangiopathies). Further studies are needed to establish its diagnostic value (specificity and sensitivity) in development of different types of vascular late complications.

In conclusion, chitotriosidase activity in plasma of patients with ongoing type 2 diabetes was significantly higher than in the control group, and was positively associated with hyperglycemia and hypertension (independently from other metabolic risk factors of diabetes progression) as well as with higher risk of occurrence of diabetic angiopathies, which indicates its potential significance in the course of type 2 diabetes.

\section{Conflict of interest}

The authors declare no conflict of interest.

\section{References}

1. Guan SP, Mok YK, Koo KN, Chu KL, Wong WS. Chitinases: biomarkers for human diseases. Protein Pept Lett 2009; 16: 490-8.

2. Hollak CE, van Weely S, van Oers MH, Aerts JM. Marked elevation of plasma chitotriosidase activity. A novel hallmark of Gaucher disease. J Clin Invest 1994; 93 : 1288-92.
3. Bouzas L, Carlos Guinarte J, Carlos Tutor J. Chitotriosidase activity in plasma and mononuclear and polymorphonuclear leukocyte populations. J Clin Lab Anal 2003; 17: 271-5.

4. van Eijk M, van Roomen CP, Renkema GH, et al. Characterization of human phagocyte-derived chitotriosidase, a component of innate immunity. Int Immunol 2005; 17 : 1505-12.

5. Korolenko TA, Cherkanova MS. Chitotriosidase of human macrophages and mammalian chitinases: biological functions and abnormalities in pathology. Vestn Ross Akad Med Nauk 2009; 11: 39-45.

6. Kanneganti M, Kamba A, Mizoguchi E. Role of chitotriosidase (chitinase 1) under normal and disease conditions. J Epithel Biol Pharmacol 2012; 5: 1-9.

7. Artieda M, Cenarro A, Gañán A, et al. Serum chitotriosidase activity is increased in subjects with atherosclerosis disease. Arterioscler Thromb Vasc Biol 2003; 23: 1645-52.

8. Paneni F, Beckman JA, Creager MA, Cosentino F. Diabetes and vascular disease: pathophysiology, clinical consequences, and medical therapy: part I. Eur Heart J 2013; 34: 2436-43.

9. Utsunomiya K. Treatment strategy for type 2 diabetes from the perspective of systemic vascular protection and insulin resistance. Vasc Health Risk Manag 2012; 8: 429-36.

10. Cicero AF, Tartagni E, Ertek S. Metformin and its clinical use: new insights for an old drug in clinical practice. Arch Med Sci 2012; 8: 907-17.

11. Guardado-Mendoza R, Prioletta A, Jiménez-Ceja LM, Sosale A, Folli $F$. The role of nateglinide and repaglinide, derivatives of meglitinide, in the treatment of type 2 diabetes mellitus. Arch Med Sci 2013; 9: 936-43.

12. Filippatos TD, Rizos EC, Gazi IF, et al. Differences in metabolic parameters and cardiovascular risk between American Diabetes Association and World Health Organization definition of impaired fasting glucose in European Caucasian subjects: a cross-sectional study. Arch Med Sci 2013; 9: 788-95.

13. Recommendation of the Polish Society of Diabetology. Clinical guidelines for management of diabetes. Diabetol Klin 2012; 1 (Suppl A): A1-54.

14. ESC/EAS Guidelines for the management of dyslipidaemias. Kardiol Pol 2011; 69 (Suppl IV): 143-200.

15. Sonmez A, Haymana C, Tapan S, et al. Chitotriosidase activity predicts endothelial dysfunction in type-2 diabetes mellitus. Endocrine 2010; 37: 455-9.

16. Matheus AS, Tannus LR, Cobas RA, Palma CC, Negrato CA, Gomes Mde B. Impact of diabetes on cardiovascular disease: an update. Int J Hypertens 2013; 2013: 653789.

17. Sheikh-Ali M, Raheja P, Borja-Hart N. Medical management and strategies to prevent coronary artery disease in patients with type 2 diabetes mellitus. Postgrad Med 2013; 125: 17-33.

18. Alanbay I, Ercan CM, Sakinci M, Coksuer H, Ozturk M, Tapan S. A macrophage activation marker chitotriosidase in women with PCOS: does low-grade chronic inflammation in PCOS relate to PCOS itself or obesity? Arch Gynecol Obstet 2012; 286: 1065-71.

19. Ramanathan R, Kohli A, Ingaramo MC, et al. Serum chitotriosidase, a putative marker of chronically activated macrophages, increases with normal aging. J Gerontol A Biol Sci Med Sci 2013; 68: 1303-10.

20. Madazli R, Kucur M, Gezer A, Isman F, Bulut B. Chitotriosidase and YKL-40 in normal and pre-eclamptic pregnancies. Int J Gynaecol Obstet 2008; 100: 239-43. 
21. Kundak AA, Tascilar ME, Abaci A, et al. Serum chitotriosidase activity: is it a new inflammatory marker in obese children? J Pediatr Endocrinol Metab 2012; 25: 63-7.

22. Kabaroğlu C, Onur E, Barutçuoğlu B, et al. Inflammatory marker levels in obese adolescents with glucose intolerance: increased chitotriosidase activity. Clin Biochem 2012; 45: 281-4.

23. Kosmala-Anderson JP, Wallace LM, Turner A, Bourne C. Self-reported effects of attending the Health Foundation's Co-Creating Health self-management programme for patients with type 2 diabetes mellitus in London, England. Arch Med Sci 2014; 10: 773-81.

24. Boot RG, van Achterberg TA, van Aken BE, et al. Strong induction of members of the chitinase family of proteins in atherosclerosis: chitotriosidase and human cartilage gp-39 expressed in lesion macrophages. Arterioscler Thromb Vasc Biol 1999; 19: 687-94. 